\title{
Neuroimaging study of partial differential equation reading in brain
}

\author{
Xiao-Dong Wang ${ }^{a}$, Ming-Tung Wang ${ }^{\mathrm{b}, *}$, Duu-Jong Lee ${ }^{\mathrm{c}}$ \\ ${ }^{a}$ Department of Thermal Science and Energy Engineering, School of Mechanical Engineering, University of Science \\ and Technology Beijing, Beijing 100083, China \\ ${ }^{\mathrm{b}}$ Department of Applied Japanese, Ming-Chuan University, De Ming Road, Gui Shan District, Taoyuan County 333, Taiwan \\ ${ }^{\mathrm{c}}$ Department of Chemical Engineering, National Taiwan University, Taipei 10617, Taiwan
}

Received 10 March 2008; accepted 10 March 2008

\begin{abstract}
Building a modeling capability for a physical phenomenon using differential equations is central to chemical engineering professionals. Using functional magnetic resonance imaging (fMRI), this work for the first time measures brain activities in partial differential equation reading and joke reading by selected professionals. Experimental results demonstrate that the equation message is first processed by the primary visual cortex (V1), and is then sent to Brodmann area 7 for information integration, and Broca's area (Brodmann area 44), Wernicke's area, and the fusiform gyrus to interpret its abstract meaning. The brain handles the differential equation as a syllabary rather than a character, and remembers the equation based on its shape rather than its identity.
\end{abstract}

(C) 2008 Taiwan Institute of Chemical Engineers. Published by Elsevier B.V. All rights reserved.

Keywords: Differential equation; Comprehension; Brain; fMRI

\section{Introduction}

Functional brain imaging and mapping have been utilized to investigate which and how brain regions are activated in response to different environmental stimuli. Kobayashi et al. (2006) investigated whether the human brain has a center for predicting the behavior of others. Crinion et al. (2006) analyzed how a bilingual brain is activated to identify and control the language in use. Sugihara et al. (2006) investigated whether humans have a universal center in the brain for writing. Certain brain regions, such as medial prefrontal cortex $(\mathrm{mPFC})$ and temporo-parietal junction (TPJ), were significantly correlated with the activity of the human "mind" (Decety and Grezes, 2006). Tsao et al. (2006) and Schwarzlose et al. (2005) determined that the fusiform face area (FFA) in the fusiform gyrus of the brain is strongly associated with face recognition.

Learning, which consists of vision, comprehension, reasoning, and information storage, is a dynamic brain process. Brain imaging research into mathematic implementation is still limited (Tang et al., 2006). Building a modeling capability for a

\footnotetext{
* Corresponding author. Tel.: +88633507001x3631; fax: +886 228331347. E-mail address: wangmt2002@yahoo.com.tw (M.-T. Wang).
}

physical phenomenon using differential equations is central to chemical engineering professionals (Arlabosse and Chitu, 2007; Hwang et al., 2006; Islam et al., 2007; Kung, 2006; Lee et al., 2006, 2007; Otawara and Kitamura, 2006; Tien and Ramarao, 2006; Seginer and Bux, 2006; Seginer et al., 2007; Tao et al., 2006). Equations have the same meaning in content comprehension and mental calculation across different cultures and languages. To the authors' best knowledge, no studies have explored how the brain processes partial differential equations during reading and comprehension. This work investigates brain activity by applying functional magnetic resonance imaging (fMRI) on the activated areas of brain and along the stream the read message is sent on in the brain. The ultimate goal of this series study is to provide information on "what works" in chemical engineering classrooms.

\section{Materials and methods}

\subsection{Subjects and tasks}

Right-handed university professors aged 32-46 years participated in this study. All subjects were native Chinese speakers with no history of neurological disorders, and had normal or corrected vision. Written consent was obtained from all subjects. 
Two block designs were utilized: (1) reading a joke silently and (2) reading the following heat conduction equation silently $\rho C_{p} \frac{\partial T}{\partial t}=k \frac{\partial^{2} T}{\partial x^{2}}+\dot{q}$. The joke in Chinese was selected with an appropriate time span allotted for reading.

\subsection{Stimuli}

The two tasks were blocked in a random order and interleaved with the fixation condition in each experimental session. For each block test, two scanning periods were utilized: (1) when one of the two tasks was performed and (2) a rest period (baseline). Each block continued for $600 \mathrm{~s}$, and a task and rest period were repeated 10 times.

Stimulus presentation was controlled by a PC. Stimuli were projected via an LCD projector onto a screen located at the subject's feet. Each subject could clearly see the screen at a viewing distance of roughly $2 \mathrm{~m}$.

\subsection{MRI procedures}

Echo planar imaging (EPI) was performed using a $3 \mathrm{~T}$ GE Sigma LX system with a standard head coil. The T2*-weighted time-series images depicting blood oxygenation level-dependent (BOLD) contrast were acquired using a gradient-echo EPI sequence $\left(\mathrm{TR}=2 \mathrm{~s} ; \mathrm{TE}=25 \mathrm{~s}\right.$; flip angle $=90^{\circ} ; \mathrm{FOV}=24 \mathrm{~cm}$ $\times 24 \mathrm{~cm}$; $64 \times 64$ matrix; in-plane resolution, $3.75 \mathrm{~mm} \times 3.75$ $\mathrm{mm}$ ). Thirty-five axial contiguous 4-mm thick slices covering the whole brain were collected. Each block for each condition lasted for 15 volumes (the instruction for $2 \mathrm{~s}=1 \mathrm{vol}$, and each task period was $30 \mathrm{~s}=15 \mathrm{vol})$. Here, 150 volumes were obtained in a single session. Structural high-resolution T1 images were also collected prior to experimental sessions $(\mathrm{TR}=10.212$; $\mathrm{TE}=4.856$; flip angle $=90^{\circ} ; \mathrm{FOV}=24 \mathrm{~cm} \times 24 \mathrm{~cm} ; 521 \times$ 512 matrix; 172 slices; 0.9-mm thick; no gap).

\subsection{Imaging data analysis}

Data were analyzed using Statistical Parametric Mapping (SPM2) (Wellcome Department of Cognitive Neurology, London), running under Matlab 7.0.0 (Mathworks, Sherbon, MA, USA). The first four scans were excluded from the analysis to minimize T1 relaxation artifacts; the remaining 146 volumes were processed. No slice timing correction was applied. All volumes were realigned to the first volume with 4th-degree Bspline interpolation. The volumes were then resliced. A mean EPI image was derived, to which the individual T1 image was coregistered. Individual $\mathrm{T} 1$ images were spatially normalized to the Montreal Neurological Institute (MNI) T1 template $(2 \mathrm{~mm} \times 2 \mathrm{~mm} \times 2 \mathrm{~mm})$ of SPM2 with medium nonlinear regularization. Transformation parameters were derived from this process, and parameters were then applied to individual $\mathrm{T} 1$ images and to all EPI images. Individual T1 images were resampled into $1 \mathrm{~mm} \times 1 \mathrm{~mm} \times 1 \mathrm{~mm}$ voxels, and EPI images were resampled into $3 \times 3 \times 3 \mathrm{~mm}^{3}$ voxels with 4 th-degree Bspline interpolation. Finally, the EPI images were smoothed with a Gaussian kernel of an $8-\mathrm{mm}$ isotropic full wave at halfmaximum.
Statistical analyses were conducted using SPM2. Functional data were evaluated as a block design using a general linear model approach. Each subject's dataset consisted of 300 volumes, which were collapsed into two conditions (i.e., task and RE) images with SPM's canonical hemodynamic response function (HRF) with a time derivative. Global scaling was not employed. High-pass temporal filtering with a cut-off of $128 \mathrm{~s}$ was applied, and serial autocorrelations were modeled with an AR(1) model in SPM2.

\section{Results and discussion}

\subsection{Joke reading}

Fig. 1(a) and Table 1 present the significant brain activation during joke reading versus fixation $(p<0.001$, uncorrected).

On joke reading, five activation areas were identified. The activation area $1 \mathrm{j}$ is composed of 1766 voxels, peaking at [27, $75,-15]$, mainly constituting left cerebrum $(38.4 \%)$ and right cerebrum (45.3\%). Detailed sub-areas were lingual gyrus [left $(12.6 \%)$ and right $(17.3 \%)$ ], middle occipital gyrus [left $(9.1 \%)$ and right $(7.3 \%)$ ], cuneus [left $(8.2 \%)$ and right $(8.3 \%)$ ], fusiform gyrus [left (3.6\%) and right (5.5\%)], inferior occipital gyrus [left (3.2\%) and right (5.3\%)], and declive [left (3.4\%) and right $(4.4 \%)]$. This activation area encompassed the Brodmann area 17 [left (3.2\%) and right (4.3\%)], 18 [left (7.8\%) and right (9.9\%)], and 19 [left (2.9\%) and right (3.1\%)].

The activation area $2 \mathrm{j}$ is composed of 43 voxels, peaking at $[-24,-60,48]$, mainly constituting left cerebrum (100\%). Detailed sub-areas were precuneus [left (34.9\%)] and superior parietal lobule [left $(65.1 \%)]$. This activation area incorporated the Brodmann area 7 [left (32.6\%)].

The activation area $3 \mathrm{j}$ is composed of 23 voxels, peaking at $[33,-75,21]$, principally constituting right cerebrum (100\%). Detailed sub-areas were sub-gyral [right (60.9\%)], middle temporal gyrus [right $(26.1 \%)$ ], and superior occipital gyrus [right (13.0\%)]. This activation area involved the Brodmann area 19 [left (4.4\%)].

The activation area $4 \mathrm{j}$ is composed of 16 voxels, peaking at $[-39,-9,42]$, mainly constituting left cerebrum (100\%). Detailed sub-area was precentral gyrus [left (100\%)]. This activation area encompassed the Brodmann area 6 [left $(37.5 \%)]$.

The activation area $5 \mathrm{j}$ is composed of 13 voxels, peaking at $[-30,-72,-3]$, mainly constituting left cerebrum (100\%). Detailed sub-area was occipital lobe [left (53.9\%)] and temporal lobe [left (7.7)]. No Brodmann area was incorporated with the activation area $5 \mathrm{j}$.

\subsection{Partial differential equation reading}

Fig. 1(b) and Table 2 present the significant brain activation during equation reading versus fixation $(p<0.001$, uncorrected).

During equation reading, 9 activation areas were identified. Activation area 1e is composed of 2240 voxels, peaking at [39, $-75,-18]$, primarily constituting the left cerebrum (35.9\%) 

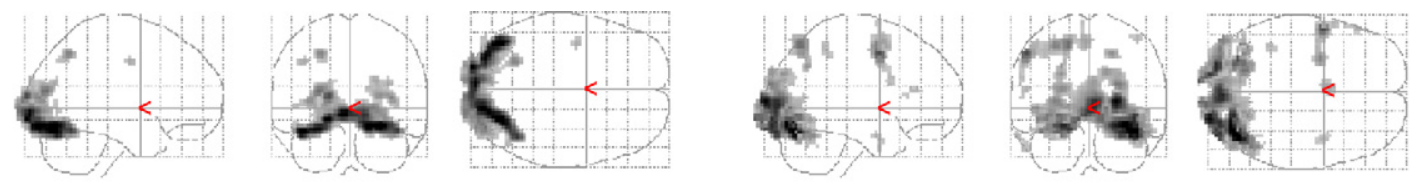

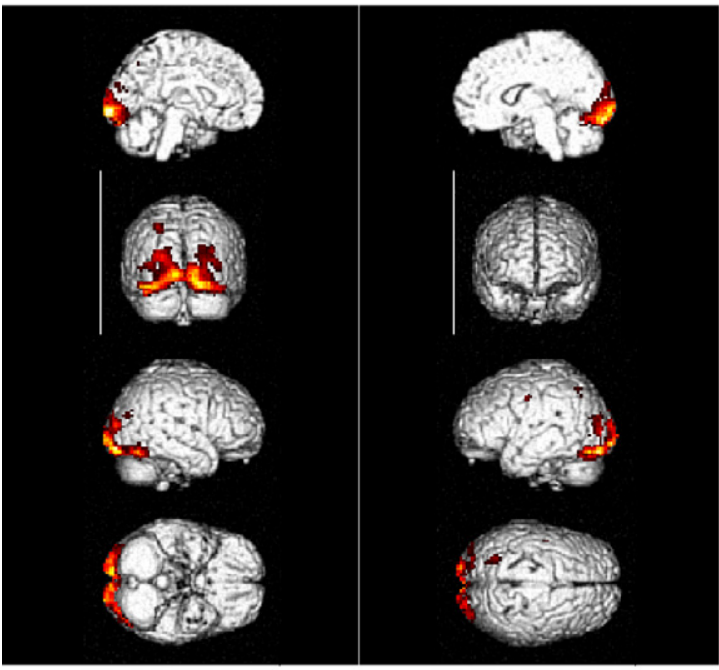

(a)

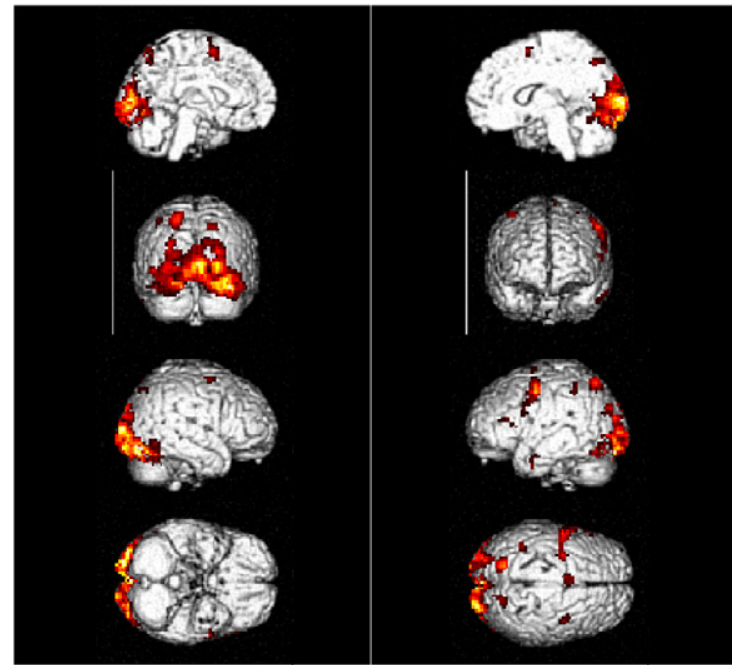

(b)

Fig. 1. Group-averaged maps for (a) joke reading and (b) equation reading relative to the fixation yielded from the scans onto a standard MNI template by SPM2. Clusters with an uncorrected $p<0.001$ with spatial extent $>20$ were shown.

and right cerebrum (51.0\%). Detailed sub-areas were sub-gyral [left $(2.41 \%)$ and right $(1.79 \%)$ ], fusiform gyrus [left $(1.21 \%)$ and right (3.93\%)], inferior occipital gyrus [left (3.75) and right (6.56)], lingual gyrus [left (9.42) and right (11.38\%)], middle occipital gyrus [left $(7.63 \%)$ and right $(11.12 \%)]$, cuneus [left $(9.60 \%)$ and right $(15.18 \%)$ ], and declive [left $(1.56 \%)$ and right (4.42\%)]. These activation areas incorporated the Brodmann areas 17 [left (2.6\%) and right (3.4\%)], 18 [left (6.7\%) and right (10.2\%)], and 19 [left (3.1\%) and right (6.5\%)].

Activation area $2 \mathrm{e}$ is composed of 161 voxels, peaking at $[-54,0,47]$, principally constituting the left cerebrum (95.7\%). Detailed sub-areas were sub-gyral [left $(1.9 \%)]$, precentral gyrus [left (39.1\%)], inferior frontal gyrus [left (8.7\%)], and middle frontal gyrus [left $(48.5 \%)]$. This activation area encompassed Brodmann areas 6 [left (39.1\%)], 8 [left (1.2\%)], 9 [left (3.7\%)], and 45 [left (1.2\%)].

Activation area $3 \mathrm{e}$ is composed of 122 voxels, peaking at $[-54,0,48]$, generally comprising the left cerebrum (75.4\%). Detailed sub-areas were the precuneus [left $(32.0 \%)$ ] and superior parietal lobule [left $(45.1 \%)]$. This activation area incorporated Brodmann area 7 [left (34.4\%)].

The activation area $4 \mathrm{e}$ is composed of 64 voxels, peaking at $[-54,0,48]$, principally the inter-hemispheric $(17.2 \%)$ and left cerebrum $(76.6 \%)$. Detailed sub-areas were superior frontal gyrus [left $(45.3 \%)$, right $(3.1 \%)$, and central $(4.7 \%)$ ] and medial frontal gyrus [left $(26.6 \%)]$. This activation area included the Brodmann areas 6 [left (43.8\%) and right (3.1\%)] and 32 [left $(3.1 \%)$ ].

The activation area $5 \mathrm{e}$ is composed of 33 voxels, peaking at $[21,-66,48]$, mainly constituting the right cerebrum (100\%). Detailed sub-areas were precuneus [right (72.3\%)] and superior parietal lobule [right (27.3\%)]. This activation area incorporated the Brodmann area 7 [left (48.5\%)].

The activation area $6 \mathrm{e}$ is composed of 22 voxels, peaking at $[-39,-45,45]$, principally constituting the left cerebrum $(100 \%)$. Detailed sub-areas were sub-gyral [left $(9.1 \%)]$ and inferior parietal lobule [left (90.9\%)]. The Brodmann area incorporated with the activation area is 40 [left (54.6\%)].

Table 1

Activation during joke reading

\begin{tabular}{|c|c|c|c|c|c|c|}
\hline \multirow[t]{2}{*}{ Activated region (approximate BA) } & \multirow[t]{2}{*}{ Left/Right } & \multicolumn{3}{|c|}{ MNI coordinates } & \multirow[t]{2}{*}{$Z$ score } & \multirow[t]{2}{*}{ Number of voxels } \\
\hline & & $x$ & $y$ & $z$ & & \\
\hline Declive & $\mathrm{R}$ & 27 & -75 & -15 & 6.94 & 1766 \\
\hline Precuneus (BA 7) & $\mathrm{L}$ & -24 & -60 & 48 & 4.54 & 43 \\
\hline Middle temporal gyrus & $\mathrm{R}$ & 33 & -75 & 21 & 3.95 & 23 \\
\hline Precentral gyrus & $\mathrm{L}$ & -39 & -9 & 42 & 3.90 & 16 \\
\hline Lingual gyrus & $\mathrm{L}$ & -30 & -72 & -3 & 3.58 & 13 \\
\hline
\end{tabular}


Table 2

Activation during equation reading

\begin{tabular}{|c|c|c|c|c|c|c|}
\hline \multirow[t]{2}{*}{ Activated region (approximate BA) } & \multirow[t]{2}{*}{ Left/Right } & \multicolumn{3}{|c|}{ MNI coordinates } & \multirow[t]{2}{*}{$Z$ score } & \multirow[t]{2}{*}{ Number of voxels } \\
\hline & & $x$ & $y$ & $z$ & & \\
\hline Declive & $\mathrm{R}$ & 39 & -75 & -18 & 7.46 & 2240 \\
\hline Frontal lobe, precentral gyrus (BA 6) & $\mathrm{L}$ & -54 & 0 & 47 & 5.83 & 161 \\
\hline Superior parietal lobule (BA 7) & $\mathrm{L}$ & -18 & -69 & 57 & 5.21 & 122 \\
\hline Medial frontal gyrus (BA 6) & $\mathrm{L}$ & -3 & 3 & 60 & 4.30 & 64 \\
\hline Precuneus (BA 7) & $\mathrm{R}$ & 21 & -66 & 48 & 4.55 & 33 \\
\hline Inferior parietal lobule (BA 40) & $\mathrm{L}$ & -39 & -45 & 45 & 3.59 & 22 \\
\hline Middle frontal gyrus (BA 6) & $\mathrm{R}$ & 42 & 0 & 60 & 3.81 & 19 \\
\hline Middle temporal gyrus & $\mathrm{L}$ & -60 & 0 & -27 & 3.91 & 15 \\
\hline Middle frontal gyrus (BA 46) & $\mathrm{L}$ & -54 & 33 & 15 & 3.89 & 15 \\
\hline
\end{tabular}

The activation area $7 \mathrm{e}$ is composed of 19 voxels, peaking at $[42,0,60]$, mainly constituting the right cerebrum $(100 \%)$. The detailed sub-area was the middle frontal gyrus [right(57.9\%)]. The Brodmann area incorporated with the activation area is 6 [right (31.6\%)].

The activation area $8 \mathrm{e}$ is composed of 15 voxels, peaking at $[42,0,60]$, principally constituting the left cerebrum (73.3\%). Detailed sub-area included inferior temporal gyrus [left (40.0\%)] and middle temporal gyrus [right (40.0\%)]. The Brodmann areas incorporated with the activation area are 20 [right (6.7\%)] and 21 [left (13.3\%)].

The activation area $9 \mathrm{e}$ is composed of 15 voxels, peaking at $[-54,33,15]$, mainly constituting the left cerebrum $(100 \%)$. Detailed sub-areas were inferior frontal gyrus [left (93.3\%)] and middle frontal gyrus [left (6.7\%)]. The Brodmann areas incorporated with the activation area are 45 [right (13.3\%)] and 46 [left (20.0\%)].

\subsection{Brain processing}

Vision studies have demonstrated that a visual signal received by the brain is first analyzed in the primary visual cortex (V1), and is then sent to relatively higher levels (V2-4) and motion-sensitive area MT through a ventral stream (to identify "what the object is") or dorsal stream (to identify "where the object is") (Driver and Mattingley, 1998; Squire et al., 2003).

Activation area $1 \mathrm{j}$ during joke reading corresponds closely with visual processing areas [V1-3] (Brodmann areas 17-19). Activation of both the left lingual gyrus and fusiform gyrus corresponds to recognition when reading a sentence or identifying an object (Tsao et al., 2006). The joke message was then sent from V1 to the left superior parietal lobule (Brodmann area 7, Somatosensory Association Cortex) via a dorsal stream for conscious awareness (Beck et al., 2001). Subsequent processing was generally weak in brain area activation; for example, the firing of the left Pre-motor and Supplementary Motor Cortex (Brodmann area 6) only obtained 7 voxels during scanning. The joke did not significantly stimulate the brains of the subjects.

Significant activation of brain areas was noticeable during equation reading tests (Fig. 1(b)). The equation message was received by $\mathrm{V} 1$ as in joke reading, with activation of the lingual gyrus and fusiform gyrus. The message was then sent through the left superior parietal lobule (Brodmann area 7 in area 3e) and middle frontal gyrus (Brodmann area 6) to the superior frontal gyrus (Brodmann areas 8 and 9) and Broca area (Brodmann area 45). Some of the message was also sent to Wernicke's area (11 voxels in Brodmann area 40) from Broca's area via the arcuate fasciculus, and to the inferior and middle temporal gyrus (Brodmann areas 20 and 21).

The brain processed equations via the dorsal stream, suggesting that the equation is interpreted by brain as a syllabary rather than as a character, and the equation is primarily identified by its shape rather than identity (Kuo et al., 2004). The Brodmann area 7 integrated the information and was the processing center for the visual message (Beck et al., 2001; Portas et al., 2000). The Broca area (Brodmann area 44) corresponding to the synthesis of an idea or speech, Wernicke area, which is responsible for word comprehension, and the fusiform gyrus, which extracts the invariant structural representation of visual words (Kuo et al., 2001, 2003), evaluated the meaning of the heat conduction equation.

\section{Conclusions}

Using differential equations to describe physical phenomena is a core capability in Chemical Engineering. This work for the first time measures brain activity in differential equation reading and joke reading by selected professionals using functional magnetic resonance imaging (fMRI). In both readings, the vision message is first analyzed in the primary visual cortex (V1), and is then sent to relatively higher levels through a dorsal stream for conscious awareness. However, the subsequent processing was generally weak in brain area activation for joke reading.

On the other hand, the equation message was received by V1 as in joke reading, with activation of the lingual gyrus and fusiform gyrus, then was sent through the left superior parietal lobule (Brodmann area 7 in area 3e) and middle frontal gyrus (Brodmann area 6) to the superior frontal gyrus (Brodmann areas 8 and 9) and Broca area (Brodmann area 45). The brain processed equations a syllabary and the equation is primarily identified by its shape rather than identity. 


\section{Acknowledgement}

This work is financially supported by National Science Council, Taiwan.

\section{References}

Arlabosse, P. and T. Chitu, "Identification of the Limiting Mechanism in Contact Drying of Agitated Sewage Sludge,” Dry. Technol., 25, 557 (2007).

Beck, D. M., G. Rees, C. D. Frith, and N. Lavie, "Neural Correlates of Change Detection and Change Blindness," Nat. Neurosci., 4, 645 (2001).

Crinion, J., R. Turner, A. Grogan, T. Hanakawa, U. Noppeney, J. T. Devlin, T. Aso, S. Urayama, H. Fukuyama, K. Stockton, K. Usui, D. W. Green, and C. J. Price, "Language Control in the Bilingual Brain," Science, 312, 1537 (2006).

Decety, J. and J. Grezes, “The Power of Simulation: Imaging One's Own and Other's Behavior,' Brain Res., 1079, 4 (2006).

Driver, J. and J. B. Mattingley, "Parietal Neglect and Visual Awareness," Nat. Neurosci., 1, 17 (1998).

Hwang, K. J., S. F. You, and T. M. Don, "Disruption Kinetics of Bacterial Cells During Purification of Poly-beta-hydroxyalkanoate Using Ultrasonication," J. Chin. Inst. Chem. Engrs., 37, 209 (2006).

Islam, M. R., K. S. Thaker, and A. S. Mujumdar, "A Diffusion Model for a Drum Dryer Subjected to Conduction, Convection, and Radiant Heat Input," Dry. Technol., 25, 1033 (2007).

Kobayashi, C., G. H. Glover, and E. Temple, "Cultural and Linguistic Influence on Neural Bases of 'Theory of Mind': An fMRI Study with Japanese Bilinguals," Brain Lang., 98, 210 (2006).

Kung, H. H., "Nanotechnology: Opportunity for Chemical Engineering," $J$. Chin. Inst. Chem. Engrs., 37, 1 (2006).

Kuo, W. J., T. C. Yeh, J. R. Duann, Y. T. Wu, L. T. Ho, D. L. Hung, D. L. Tseng, and J. C. Hsieh, "A Left-lateralized Network for Reading Chinese Words: A 3T fMRI Study," NeuroReport, 12, 3997 (2001).

Kuo, W. J., T. C. Yeh, C. Y. Wu, Y. T. Chou, L. T. Ho, D. L. Hung, O. J. L. Tzeng, and J. C. Hsieh, "Frequency Effects of Chinese Character Processing in the Bra: An Event-related fMRI Study," Neuroimage, 18, 720 (2003).
Kuo, W. J., T. C. Yeh, J. R. Lee, L. F. Chen, P. L. Lee, S. S. Chen, L. T. Ho, D. L. Hung, O. J. L. Tzeng, and J. C. Hsieh, "Orthographic and Phonological Processing of Chinese Characters: An fMRI Study," Neuroimage, 21, 1721 (2004).

Lee, D. J., J. Y. Lai, and A. S. Mujumdar, "Moisture Distribution and Dewatering Efficiency for Wet Materials," Dry. Technol., 24, 1201 (2006).

Lee, J. E., J. K. Lee, and H. K. Choi, "Filter Press for Electrodewatering of Waterworks Sludge," Dry. Technol., 25, 1649 (2007).

Otawara, K. and T. Kitamura, "Comparison of a Belt-Filter and Centrifuge in the Crystal Purification Process with CDC (Cooling Disc Crystallizer) and KCP (Kureha Crystal Purifier)," J. Chin. Inst. Chem. Engrs., 37, 509 (2006).

Portas, C. M., K. Krakow, P. Allen, O. Josephs, J. L. Armony, and C. D. Frith, "Auditory Processing Across the Sleep-Wake Cycle: Simultaneous EEG and fMRI Monitoring in Humans," Neuron, 28, 991 (2000).

Schwarzlose, R. F., C. I. Baker, and N. Kanwisher, "Separate Face and Body Selectivity on the Fusiform Gyrus," J. Neurosci., 25, 11055 (2005).

Seginer, I. and M. Bux, "Modeling Solar Drying Rate of Wastewater Sludge," Dry. Technol., 24, 1353 (2006).

Seginer, I., I. Ioslovich, and M. Bux, "Optimal Control of Solar Sludge Dryer," Dry. Technol., 25, 401 (2007).

Squire, L. R., F. E. Bloom, S. K. McConnell, L. L. Roberts, N. C. Spitzer, and M. J. Zigmond (Eds.), Fundamental Neuroscience, 2nd Ed., Elsevier Academic Press, San Diego, U.S.A. (2003).

Sugihara, G., T. Kaminaga, and M. Sugishita, "Interindividual Uniformity and Variety of the "Writing Center": A Functional MRI Study," Neuroimage, 32, 1837 (2006).

Tang, Y. Y., W. T. Zhang, K. W. Chen, S. G. Feng, J. Ye, J. X. Shen, E. M. Reiman, and Y. J. Liu, "Arithmetic Processing in the Brain Shaped by Cultures," Proc. Natl. Acad. Sci. U.S.A., 103, 10775 (2006).

Tao, T., X. F. Peng, and D. J. Lee, "Interaction between Ice and Floc under Freezing," J. Chin. Inst. Chem. Engrs., 37, 299 (2006).

Tien, C. and B. V. Ramarao, "On Analysis of Cake Formation and Growth in Cake Filtration,” J. Chin. Inst. Chem. Engrs., 37, 81 (2006).

Tsao, D. Y., W. A. Freiwald, R. B. Tootell, and M. S. Livingstone, "A Cortical Region Consisting Entirely of Face-Selective Cells," Science, 311, 670 (2006). 